\title{
Rursus
}

Russus

Poiétique, réception et réécriture des textes antiques

10 | 2017

Traductions latines de textes grecs

\section{Traduzione ed etica nel Ludus septem sapientum di Ausonio. Un esercizio per la scuola}

Translation and Ethics in Ausonius' Ludus septem sapientum. An exercise for the school

\section{Elena Cazzuffi}

\section{OpenEdition}

\section{Journals}

Edizione digitale

URL: http://journals.openedition.org/rursus/1174

DOI: $10.4000 /$ rursus. 1174

ISSN: 1951-669X

\section{Editore}

Université Nice-Sophia Antipolis

Notizia bibliografica digitale

Elena Cazzuffi, «Traduzione ed etica nel Ludus septem sapientum di Ausonio. Un esercizio per la scuola », Rursus [En ligne], 10 | 2017, mis en ligne le 28 août 2017, consulté le 30 avril 2019. URL http://journals.openedition.org/rursus/1174; DOI : 10.4000/rursus.1174

Questo documento è stato generato automaticamente il 30 aprile 2019.

Rursus 


\section{Traduzione ed etica nel Ludus septem sapientum di Ausonio. Un esercizio per la scuola ${ }^{1}$}

Translation and Ethics in Ausonius' Ludus septem sapientum. An exercise for the school

Elena Cazzuffi

\section{Lingua e cultura greca nel Ludus}

1 Molto è stato scritto sul rapporto di Ausonio con la lingua greca e, per sintetizzare i risultati dei moderni studi sul tema, è sufficiente riferire che, al di là di topiche asserzioni di inadeguatezza ${ }^{2}$, dagli Opuscula emerge una notevole competenza: il bordolese non fu solo lettore ${ }^{3} \mathrm{e}$ traduttore dal greco ${ }^{4}$, ma anche autore creativo, come dimostrano i giochi di Sprachmischung dell'epistolario ${ }^{5}$. Sicuramente il greco non era estraneo al contesto familiare ${ }^{6}$ del poeta e, d'altra parte, per un docente ${ }^{7}$ era indispensabile averne dimestichezza; infatti nella Gallia del IV secolo il piano di studi prevedeva ancora l'insegnamento della seconda lingua ${ }^{8}$, che tuttavia doveva essere appresa con risultati soddisfacenti solo da un piccola percentuale di allievi ${ }^{9}$.

L'indagine sul greco nella scuola, nel tardoantico e in area periferica è stata documentata facendo ricorso ai numerosi componimenti ausoniani in cui è più o meno evidente una relazione con l'idioma e con il sapere ellenico; tra essi figura un opusculum talvolta sottovalutato dalla critica ${ }^{10}$, s'intende il Ludus septem sapientum, che ha il pregio di offrire una panoramica completa su questioni dibattute, quali la traduzione e il confronto tra le culture greca e romana. All'epistola prefatoria in distici elegiaci, indirizzata a Latino Pacato Drepanio ${ }^{11}$, seguono nove monologhi in senari giambici pronunciati da un prologus, da un ludius e dai sette sapienti, Solone, Chilone, Cleobulo, Talete, Biante, Pittaco e Periandro; i saggi si susseguono sul palco riproducendo convenzioni del teatro romano, 
per presentare in greco, tradurre in latino e commentare le $\gamma v \tilde{\omega} \mu \alpha l$ che tradizionalmente sono loro attribuite.

3 Il Ludus ha un chiaro intento pedagogico e un impianto mnemotecnico, ma veicola, oltre alle nozioni ${ }^{12}$, anche insegnamenti morali, secondo un uso invalso nelle classi di grammatica e di retorica ${ }^{13}$ e testimoniato da altri opuscula di contenuto filosofico (quali le egloghe 19-21) ${ }^{14}$. SIVAN 1993 (77), sulla scorta di GREEN 1991 (596), ha confermato la destinazione scolastica dell'opera individuando proprio nell'insegnamento del greco il suo obiettivo didattico. Indubbiamente si nota una generale adesione alle "proposte educative" della scuola romana, ma pare che l'avviamento alla seconda lingua non sia la sua principale mira perché il testo greco, ridotto alle sole $\gamma v \tilde{\omega} \mu \alpha$ e quindi a una configurazione immutabile, non può offrire fondamenti grammaticali, né morfologici né sintattici; piuttosto le massime tradotte potevano servire per la comparazione linguistica e per la riflessione etica e forse costituire una premessa ai primi studi filosofici ${ }^{15}$. Infatti, nei loro monologhi i sapienti traggono spunto dalla presentazione e dalla traduzione latina dei dicta per sviluppare un'analisi critica del contenuto morale trasmesso da ciascun enunciato, spesso mettendo a confronto la sapienzialità ellenica con quella romana.

\section{Il Ludus nel panorama delle composizioni catalogiche greche e latine sui sette sapienti}

4 Prima di esaminare puntualmente le traduzioni presenti nel Ludus, è utile un preliminare confronto con il corpus dei componimenti greci e latini incentrati sui sette savi ${ }^{16}$.

Ci è nota una serie omogenea di compilazioni metriche in cui, nel volgere di pochi versi, si enunciano i nomi dei sapienti, corredati dalle rispettive provenienze, insieme alle loro massime (in genere si tratta della $\gamma v \omega ́ \mu \eta$ che apre la rubrica di ciascun saggio nella

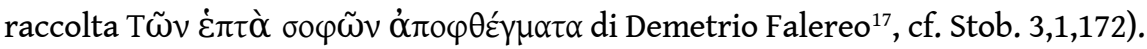

Sul versante greco si ricordano Anth. Pal. 7,81 (Antipatro di Sidone?, II sec. a.C.) ${ }^{18}$ e Anth. Pal. 9,366 ${ }^{19}$. Per il settore latino i sette esametri di Hyg. fab. 221 e i sette distici elegiaci di Lux. anth. 351Riese ${ }^{2}$ rappresentano cronologicamente il primo e l'ultimo saggio di elenco poetico a tematica sapienziale; a questi vanno aggiunte le sequenze di Sidon. carm . 2,156-163 (sette esametri), di carm. 15,42-50 (sette esametri) ${ }^{20}$ e di $\mathbf{2 3 , 1 0 1 - 1 1 0 ~ ( d i e c i ~}$ endecasillabi falecei, in cui si menzionano solo i nomi con la patria, senza le massime) ${ }^{21}$ che, pur non essendo composizioni autonome, presentano le medesime caratteristiche degli elenchi sopra citati.

Questi "inventari" poetici riflettono la cultura e il gusto di una società aggrappata alla tradizione retorica e scolastica e, per questo, propensa alla catalogazione e alla diffusione di un sapere enciclopedico, e, certamente, pratica dell'esercizio versificatorio. Anche il Ludus risente delle medesime sollecitazioni, tuttavia Ausonio rifiuta la regolarità dei componimenti citati: il numero di versi destinato ai savi varia in modo considerevole in rapporto al personaggio, più o meno noto, e alla ricchezza del suo monologo, a seconda che sia corredato di aneddoti, di indicazioni per la realizzazione pratica della sentenza o di raffronti con la cultura romana; le $\gamma v \tilde{\omega} \mu \alpha$ e i dicta, poi, compaiono ripetuti fino a cinque volte all'interno del poemetto ${ }^{22}$, verosimilmente alla scopo di facilitarne la memorizzazione; infine il Ludus è l'unica composizione latina a presentare le massime utraque lingua. 


\section{La traduzione delle $ү v \tilde{\omega} \mu a \iota$ come premessa ai contenuti etici}

8 Secondo un uso consolidato nei prologhi della commedia arcaica, cui il Ludus continuamente si richiama ${ }^{23}$, un Ludius, anticipa il contenuto dell'opera indicando uno per uno i nomi dei savi e le loro massime. Come Plauto e Terenzio segnalavano il titolo del modello greco a cui si rifacevano, dandone una traduzione e talvolta motivando la scelta di una diversa intestazione, a sua volta Ausonio interviene a spiegare le ragioni della propria versione.

(51) Delphis Solonem scripse fama est Atticum

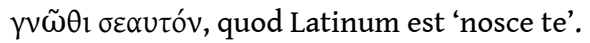

Multi hoc Laconis esse Chilonis putant.

(55) Spartane Chilon, sit tuum necne ambigunt,

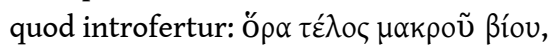

finem intueri longae vitae qui iubes.

Multi hoc Solonem dixe Croeso existimant.

Et Pittacum dixisse fama est Lesbium

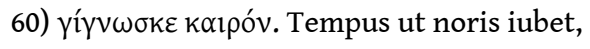

sed kaıpó iste tempestivum tempus est.

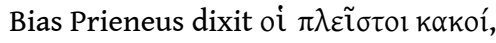

quod est Latinum 'plures hominum sunt mali'.

Sed imperitos scite quos dixit malos.

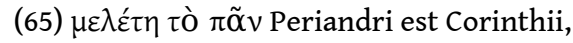

meditationem esse totum qui putat.

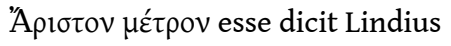

Cleobulus, hoc est 'optimus cunctis modus'.

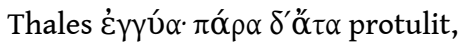

(70) spondere qui nos, noxa quia praesto est, vetat.

Hoc nos monere faeneratis non placet.

Dixi; recedam. Legifer venit Solon.

(lud. 52-68) 24 $^{24}$

Il Ludius segnala quando non vi è una perfetta sovrapposizione semantica tra i due idiomi,

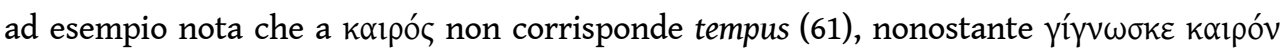
sia tradotto con tempus ut noris iubet (60), e precisa: kaıрós iste tempestivum tempus est (60), avvertendo quasi una lacuna nelle possibilità espressive del latino; nel suo monologo, poi, Pittaco ricorda anche un corrispettivo latino: Romana sic est vox, 'venite in tempore' (lud. 206), citazione di Ter. Andr. 758. Ancora, il plures hominum sunt mali, dal greco oi $\pi \lambda \varepsilon \tilde{\tau} \sigma \tau \mathrm{t}$ кরkoí (lud. 62s.), è accompagnato dall'interpretazione di mali come imperiti, significato estraneo all'etica aristocratica della Grecia arcaica.

10 Nei successivi monologhi la versione dal greco costituisce il presupposto indispensabile alla riflessione sulla $\gamma v \omega ́ \mu \eta$ stessa; la resa latina insiste in qualche caso su fatti linguistici, spesso su aspetti culturali e sempre fa attenzione al messaggio etico con inviti a una pratica di vita controllata e diretta sotto la guida di principi tradizionali. Le traduzioni si lasciano ricondurre tutte alla seguente casistica:

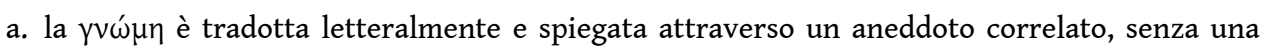
sovrapposizione culturale romana;

b. la $\gamma v \omega ́ \mu \eta$ è tradotta senza precisazioni linguistiche, ma è interpretata alla luce del costume romano; 
c. la traduzione comprende una citazione letteraria e l'interpretazione è condotta secondo il pensiero romano tradizionale;

d. la versione è letterale, ma richiede precisazioni linguistiche ed è corredata di una interpretatio romana.

11 Spesso compaiono alcune espressioni-spia a introdurre la resa latina: quod Latinum est (v. $53)^{25}$ e quod est Latinum (v. 63), hoc est (v. 68), si Latine edisseras (v. 86), interpretare tu (150) ${ }^{26}$, Latinum est (v. 181), Latine dictum suspicor (v. 190), quod vocant (v. 205), Romana sic est vox (v. 206); alcune marche affiancano anche il greco: Graece coactum est (v. 85), Graece dicimus (v. 180).

\section{a. Traduzione letterale, priva di adattamenti}

12 La prima tipologia traduttiva è rappresentata nel monologo soloniano:

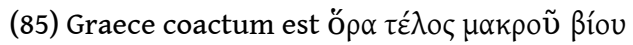

quod longius fit, si Latine edisseras.

Spectare vitae iubeo cunctos terminum;

proinde miseros aut beatos dicier,

eventa quod sunt semper ancipiti statu.

(lud. 85-89)

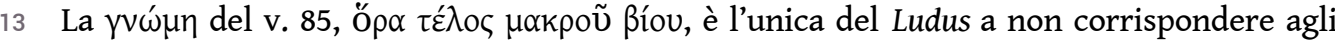
elenchi di Demetrio Falereo (vd. Stob. 3,1,172) che ricorda per prima tra le sentenze pronunciate da Solone il $\mu \eta \delta \grave{v} v$ ó $\gamma \alpha v$ (qui ascritto a Cleobulo, v. 156); l'adozione di questa massima risponde all'esigenza narrativa di presentare la seguente versione latina dell'incontro tra il legislatore ateniese e il re lido Creso (vv. 91-125) ${ }^{27}$.

14 Al greco, più conciso (coactum est) ${ }^{28}$, è accostato il latino spectare vitae iubeo cunctos terminum, più esteso (longius fit), nonostante l'omissione di un corrispettivo di $\mu \alpha \kappa \rho \circ \tilde{v}^{29}, \mathrm{e}$ precisato dall'enunciato del v. 88 e dall'ulteriore sentenza del v. 89: eventa quod sunt semper ancipiti statu ${ }^{30}$, che figurava già in Val. Max. 7,2ext.2 quale corollario della $\gamma v \omega ́ \mu \eta$ soloniana (age, quam prudenter Solo neminem, dum adhuc viveret, beatum dici debere arbitrabatur, quod ad ultimum usque fati diem ancipiti fortunae subiecti essemus) ed era a sua

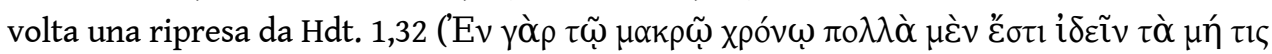

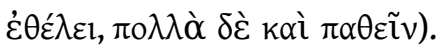

15 Al v. 86 il verbo edissero, che propriamente non significa 'tradurre' ${ }^{31}$, getta luce sul valore delle versioni nel Ludus: non si tratterebbe di semplici trasposizioni da una lingua all'altra, ma di articolate "interpretazioni" dal greco; tant'è che in precendenza il poeta era ricorso alla sostantivazione dell'intensivo di edissero, edisserto, per precisare la funzione del Ludius, definito al v. 51 edissertator ${ }^{32}$ perché a lui è affidato il compito di anticipare le massime in greco e in latino.

\section{b. Traduzione letterale, con interpretatio romana}

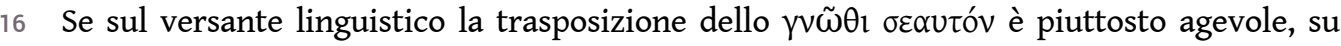
quello etico-pratico la $\gamma v \omega ́ \mu \eta$ subisce un radicale adattamento rispetto al contesto della Grecia arcaica, in seno alla quale essa era stata elaborata:

Brevitate nota, qua Lacones utimur,

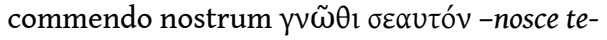

quod in columna iam tenetur Delphica. 
(140) Labor molestus iste fructi est optimi, quid ferre possis quidve non dinoscere, noctu diuque quae geras, quae gesseris, ad usque puncti tenuis instar quaerere. Officia cuncta, pudor, honor, constantia (145) in hoc et illa spreta nobis gloria. (lud. 137-145)

Nel IV secolo il nosce te ipsum era in pieno revival, era noto e sfruttato dagli autori cristiani in Oriente, dove venne rivalutato dai Padri Cappadoci, e in Occidente, dove pure fu riconsiderato in una prospettiva di fede da Ambrogio e da Agostino ${ }^{33}$; esso conobbe una certa diffusione anche in ambito pagano dove, tuttavia, cristallizzato nella rosa delle massime sapienziali, stentava ad avere una propria autonomia. Anche nel Ludus il dictum subisce un certo appiattimento: la profondità del dettato delfico, arricchita nei secoli da tanta riflessione filosofica e non, si riduce a un invito alla pratica quotidiana dell'esame di coscienza, un esercizio che Ausonio stesso praticava ${ }^{34}$. Com'è noto, la recognitio sui aveva avuto molta fortuna nel mondo romano: ce lo testimoniano Hor. serm. 1,3,34ss. 1,4,129ss. - 1,6,65ss. e, soprattutto, Sen. dial. 5,36,1-4 (=de ira 3,36,1-4); e, di conseguenza, il contesto di situazioni a cui Chilone lo riferisce è assolutamente romano: le attività e $\mathrm{i}$ valori da passare in rassegna includono gli officia (i doveri pubblici e morali), il pudor (il sentimento di appropriatezza e di decenza), l'honor (la dignità che proviene dal rango), la constantia (la risoluzione, la fermezza di propositi), la gloria (il riconoscimento e la distinzione che provengono da un'azione encomiabile).

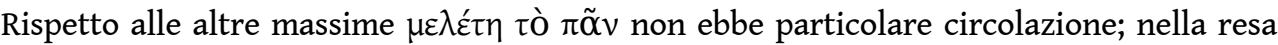
latina proposta da Ausonio vi è un aumento di materiale linguistico ( con totum quod geras), che dipende dall'esegesi della $\gamma v \omega ́ \mu \eta$, la cui applicazione è circoscritta all'ambito dell'attività pratica (negotium, v. 218) ${ }^{35}$.

Ephyra creatus huc Periander prodeo,

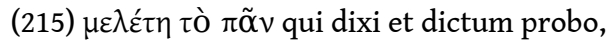

meditationem esse totum quod geras.

Is quippe solus rei gerendae est efficax,

meditatur omne qui prius negotium.

Adversa rerum vel secunda praedicat

(220) meditanda cunctis comicus Terentius.

Sedes locare, bellum gerere aut ponere,

magnas modicasque res, etiam parvas quoque

agere volentem semper meditari decet.

Nam segniores omnes in coeptis novis,

(225) meditatio si rei gerendae defuit.

Nil est quod ampliorem curam postulet

quam cogitare quid gerendum sit. Dehinc

incogitantes fors, non consilium, regit.

(lud. 214-228) ${ }^{36}$

Ogni azione si compone di una fase teorica, la meditatio, e di una fase esecutiva, la res gerenda; se viene meno la componente preliminare, il risultato dell'impresa non è garantito perché è lasciato al caso (fors, v. 228) e non è guidato dalla decisione (consilium, v. 228); questo principio è valido in ogni circostanza (adversa vel secunda, v. 219) e in ogni sorta di occupazione (v. 222), tuttavia una riflessione preparatoria è assolutamente indispensabile nel caso in cui si debbano intraprendere compiti nuovi, mai esperiti prima $(\mathrm{vv} .224 \mathrm{~s} .)^{37}$. La validità della massima è dimostrata non solo tramite un elenco di attività che ne prevedano l'impiego (vv. 221ss.), ma anche attraverso la menzione di una auctoritas 
indiscutibile, fonte a sua volta di detti per gnomologi, vale a dire Terenzio; i vv. 219s. ricordano dichiaratamente una sentenza di Phorm. 241ss.:

quam ob rem omnis, quom secundae res sunt maxume, tum maxume meditari secum oportet quo pacto advorsam aerumnam ferant, pericla damna exsilia: peregre rediens semper cogitet.

Instaurando un parallelismo tra la sapienza greca arcaica e gli enunciati sentenziosi della commedia, Ausonio pone sullo stesso piano il sapere tradizionale greco e quello romano e, di conseguenza, rivendica alla latinità una propria autonomia, acquisita già in epoca arcaica alla pari con il mondo greco arcaico.

\section{c. Traduzione mediante citazione letteraria, con adattamento al pensiero e al costume romano}

21 Tra i sapienti del Ludus Cleobulo, per primo, riconosce al mondo romano una certa

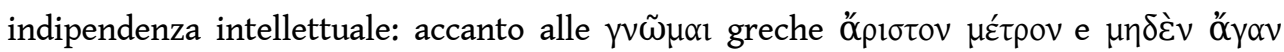
figurano le latine optimus modus ${ }^{38}$ e ne quid nimis, note sentenze del teatro arcaico (Plaut. Poen. 238 modus omnibus rebus, soror, optimumst habitu e Ter. Andr. 62 adprime in vita esse utile, ut nequid nimis).

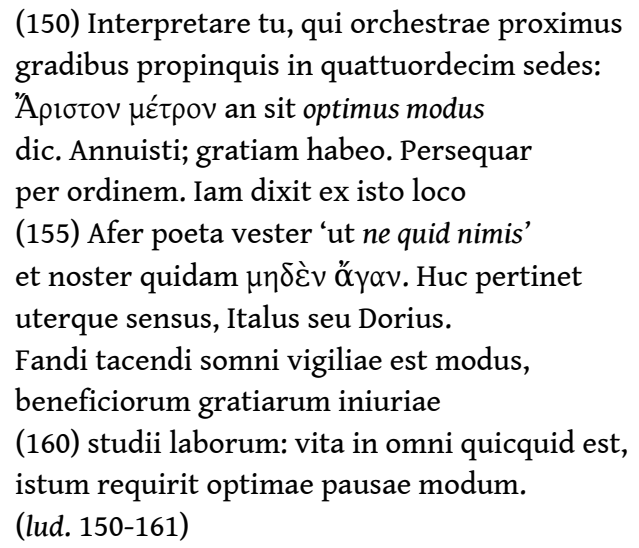

Nel presentare il secondo binomio il savio antepone la massima latina a quella greca e tiene ben distinte le rispettive provenienze mediante i possessivi vester (155) e noster (156); inoltre Cleobulo precisa che gli enunciati hanno il medesimo significato (156s.), puntualizzazione che non avrebbe senso se una sentenza fosse traduzione dell'altra. In questo modo Ausonio rileva quello che i moderni studiosi hanno affermato raccogliendo prove e attestazioni, cioè che l'optimus modus non è la trasposizione di un concetto greco, ma una nozione genuinamente romana ${ }^{39}$ : il quadro ricostruibile attraverso le fonti letterarie distingue tra la fase arcaica, in cui il modus era un valore pragmaticoutilitaristico legato al mondo rurale e immune da speculazioni greche, e la fase classica, quando i romani riconobbero nella moderazione e nell'autocontrollo il segreto dei loro continui successi militari e politici ${ }^{40}$.

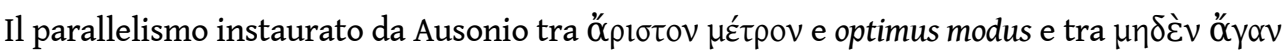
e nihil nimis e il conseguente rapporto di corrispondenza tra le quattro massime, desunto forse da uno gnomologio, fa ordine in una tradizione piuttosto caotica nella quale non è possibile capire fino a che punto le varie formulazioni del dictum siano sovrapponibili: si può concludere solamente che tutte si lasciano ricondurre a un medesimo concetto di misura. Che il contesto di riferimento sia tutto romano è confermato dalla sequenza di 
ambiti a cui estendere l'esercizio della moderazione (vv. 157-160), che, infatti, evoca altri cataloghi della letteratura arcaica, inerenti al modus:

Ennio ann. 279-285 Skutsch

... doctus, fidelis

suavis homo, iucundus, suo contentus, beatus

scitus, secunda loquens in tempore, commodus, verbum

paucum, multa tenens antiqua ...

$[\ldots]$

prudentem qui dicta loquive tacereve posset;

Lucil. frg. 1331 Marx = 1347ss. Krenkel

virtusque quaerendae finem re scire modumque,

virtus divitiis pretium persolvere posse,

virtus id dare quod re ipsa debetur honori;

e, al contrario:

Plaut. Bacch. 612ss.

petulans, protervo, iracundo animo, indomito, incogitato,

sine modo et modestia sum, sine bono iure atque honore

(cf. anche Merc. 25-31).

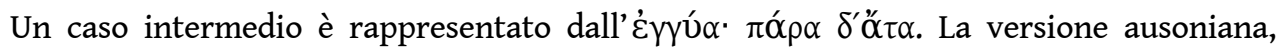
sponde; noxa <sed > praesto tibi (v. 181), si discosta in maniera evidente dalle rese delle sequenze catalogiche di Igino e di Sidonio ${ }^{41}$ e sembra ispirarsi a Ter. Phorm. 266s. hic in noxast, ille ad defendundam causam adest; / quom illest, hic praestost: tradunt operas mutuas. Il riferimento al passo terenziano, fortemente permeato dal lessico legale ${ }^{42}$, ben si presta a innescare la successiva interpretazione della $\gamma v \omega ́ \mu \eta$ in chiave giuridica romana; non a caso la discussione della massima è disseminata di termini provenienti dalla giurisprudenza (adsertor 177, sponde e noxa 181, praedes vadesque paenitudinis reos 183, nominatim dicere 184), palesando che l'interpretazione ausoniana è orientata in senso strettamente tecnico e giuridico:

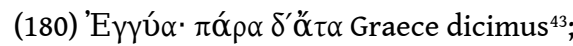

Latinum est 'sponde; noxa <sed> praesto tibi'.

Per mille possem currere exempla ut probem

praedes vadesque paenitudinis reos,

sed nolo nominatim quemquam dicere.

(lud. 180-184)

Si costituisce così intorno al dictum un retroscena latino: la cosiddetta questione legale della sponsio, una delle più antiche forme di contratto romano, usata per concludere paci e alleanze e, nel diritto familiare, per promettere una figlia in matrimonio (cf. Varro ling. 6,74 e Phaedr. 1,16).

\section{d. Traduzione letterale, con precisazioni linguistiche e interpretatio romana}

Il bianteo oi $\pi \lambda \varepsilon \tilde{\sigma} \sigma \tau o l$ kakoí doveva valere originariamente come affermazione generica "i più sono malvagi" e in questo senso lo intendono $\mathrm{i}$ componimenti catalogici latini ${ }^{44}$.

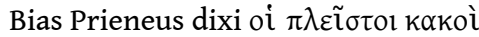

(190) Latine dictum suspicor 'plures mali'.

Dixisse nollem: veritas odium parit.

Malos sed imperitos dixi et barbaros,

qui ius <et> aequum <et> sacros mores neglegunt.

(lud. 189-193) 

più vicina al greco; tuttavia l'interpretatio che segue è radicalmente innovativa rispetto

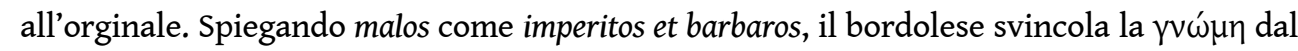
contesto dell'है $\theta$ oৎ aristocratico cui originarimente si riferiva. L'uso greco, registrato dal TLG, consente un'interpretazione di kakó come imperitus solamente quando ci si riferisca

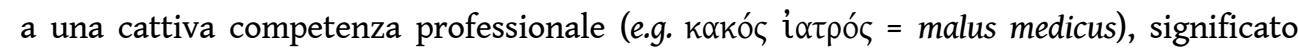
poco pertinente rispetto ai detti dei savi. D'altra parte in latino l'imperitia è di frequente un malum causa di altri mali, così in Sen. epist. 31,6 quid malum est? rerum imperitia, in epist. 76,34 magna autem pars est apud imperitos mali novitas e in epist. 78,10 illud autem est, quod imperitos in vexatione corporis male habet; ma, a questo proposito, pare appropriato menzionare soprattutto la puntualizzazione di Grill. rhet. 1,3 imperitus omnis malus est, quia, quid sit iniustum, ignorat, ut Terentius "homine imperito numquam quicquam iniustiust", con riferimento ad Ad. 98 (all'opposto, in un certo numero di luoghi terenziani bonus acquisisce il valore di "esperto", "capace”, vd. FoCARDI 1972: 73).

La seconda precisazione di malus come barbarus è ancora un'elaborazione ausoniana finalizzata all'adattamento romano. Che tale fosse il valore originario della massima greca è assolutamente da escludere perché le speculazioni sulla barbarie nel mondo greco risalgono alle guerre persiane ${ }^{45}$. L'accostamento di imperitus a barbarus non è raro nella letteratura latina ${ }^{46}$, mentre è infrequente l'uso ravvicinato con malus, implicita componente semantica di barbarus ${ }^{47}$. La chiosa è ulteriormente puntualizzata nel v. 193 qui ius <et> aequum <et> sacros mores neglegunt, che condensa in poche parole l'insieme dei valori normativi che governano il comportamento civile e morale dei romani: il diritto (lo ius è il complesso di regole comportamentali spesso a carattere consuetudinario) ${ }^{48}$, la giustizia (l'aequitas, vale a dire la ponderazione nell'applicazione della norma) e i sacri mores (garantiti dallo ius e a loro volta origine del diritto).

Questa interpretazione della massima sembra aderire alla tripartizione ciceroniana dell'umanità nelle tre categorie di romani, greci e barbari (FREYBURGER 1977: 141-152), conducendo all'identificazione dei mali con i nemici esterni e dei boni con il popolo romano seduto a teatro (cf. v. 22 togate Romule). La rassicurante ripartizione viene repentinamente scombinata nei versi successivi, significativamente introdotti dall'avversativa sed (197) che interrompe il ragionamento sulla barbarie e introduce una sorta di falso sillogismo volto a confutare la veridicità dell'apoftegma:

Sed nemo quisquam tam malus iudex fuat

quin iam bonorum partibus se copulet.

Sive ille vere bonus est seu dici studet,

(200) iam fugit illud nomen invisum mali.

Abeo. Valete et plaudite, plures boni.

(lud. 197-201)

31 L'ironico invito a non essere con se stessi giudici tanto malvagi da non accludersi alla schiera dei buoni suona più come una disillusa constatazione che induce il sapiente a smentire l'esistenza stessa del nomen mali. Infatti, l'implicazione finale del "sillogismo" nega non solo che i più siano malvagi, ma addirittura che i malvagi esistano; di qui il ridimensionamento, ancora in tono polemico, della levatura morale dei presenti: da populus ... totus bonorum (vv. 194s.) a plures boni (201) che traduce e rovescia la $\gamma v \omega ́ \mu \eta$ come enunciata nel senario iniziale del monologo ${ }^{49}$.

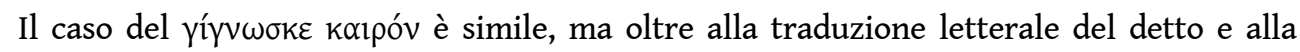
precisazione linguistica, compare un'altra citazione terenziana: 
Mitylena ortus Pittacus sum Lesbius ${ }^{50}$,

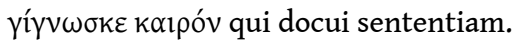

Sed iste kalpós, tempus ut noris, monet

(205) et esse kaıpóv tempestivum quod vocant

Romana sic est vox, 'venite in tempore'.

Vester quoque ille comicus Terentius

rerum omnium esse primum tempus autumat,

ad Antiphilam quom venerat servus Dromo

(210) nullo impeditam, temporis servans vicem.

(lud. 202-210)

\section{(1)}

endo l'affinità tra i due termini (v. 204), preferisce precisare il concetto di tempus (su cui vd. TRAINA 1986: 243ss.) mediante l'aggettivo tempestivus (v. 205)53; qui glossa sia il greco koıрóv che il latino tempus, evidentemente non più perspicuo nel suo significato specifico di "tempo opportuno", come dimostra la figura etimologica tempestivum tempus $(61)^{54}$; mentre, di fatto, la nozione di "circostanza appropriata, momento favorevole" era originariamente insita, oltre che in kaıpós, anche in tempus (Spitzer 2006: 91), come risulta dall'endiadi plautina occasio et tempus e dalle numerose forme avverbiali impiegate dal sarsinate con riferimento a quella nozione: in tempore, per tempus, temperi, tempore, ad tempus, ex tempore, in tempore, in tempus, per tempus e pro tempore ${ }^{55}$.

\section{Greco e latino: unità della riflessione classica e delle sue categorie di giudizio}

Le traduzioni proposte nel Ludus sono tutte anticipate da un "code-switching" ${ }^{56}$, necessario ogni qual volta venga presentata una $\gamma v \tilde{\omega} \mu \eta$. Riferiamo, in quanto rappresentativi dei fenomeni dell'intero poemetto, i casi di commistione linguistica ${ }^{57}$ della sezione del Ludius: talvolta l'amalgama tra le due lingue è più profonda perché si verifica entro i confini della frase e dà luogo a una vera e propria fusione sintattica

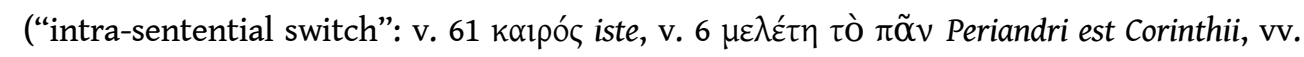

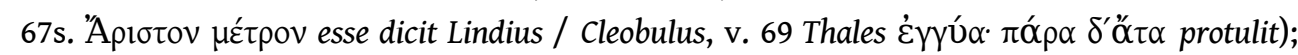
talvolta latino e greco rispettano ciascuno i confini del proprio enunciato ("inter-

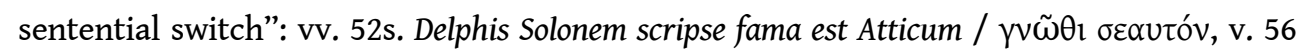

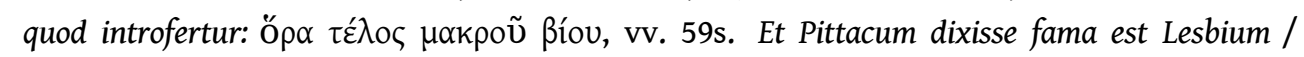

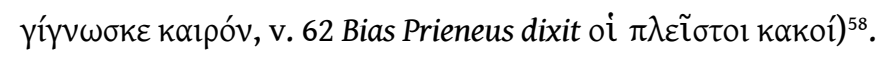

Al "cambio di codice" segue sempre una traduzione del greco proposta in modi differenti. Per citare ancora la sezione del Ludius, troviamo traduzioni nettamente riconoscibili (e.g. v. 53 ... quod est Latinum 'nosce te', v. 63 quod est Latinum 'plures hominum sunt mali', v. 68 ... hoc est 'optimus cunctis modus') e altre che, prive di una marca introduttiva e non sempre 
puntualmente coincidenti con il greco, sembrano piuttosto delle prescrizioni (v. 57 finem intueri longae vitae qui iubes, v. 60 ... tempus ut noris iubet, v. 70 spondere qui nos, noxa quia praesto est, vetat); altre traduzioni sono chiose ideate per puntualizzare un concetto (v. 61 sed kaıpó iste tempestivum tempus est, v. 64 sed imperitos scite quos dixit malos; si noti che le precisazioni semantiche sono tutte introdotte dall'avversativa: cf. anche v. 192 malos sed imperitos dixi et barbaros e 204s. sed iste kaıpó ... monet / et esse kaıpóv tempestivum quod vocant). In ogni caso, benché si tratti di vere e proprie versioni, Ausonio non insiste sull'importanza della traduzione (come si evince dall'assenza di una terminologia del tradurre, cf. nota 25) e piuttosto cerca di eludere la giustapposizione degli enunciati greci e latini, sia visivamente (evitando di segmentarli in versi differenti) sia concettualmente (presentando le traduzioni alla pari con l'originale). Poiché si tratta con buona probabilità di un testo pensato originariamente per la scuola, potrebbe stupire il fatto che il maestro Ausonio non focalizzi proprio sulla metodologia di traduzione ${ }^{59} \mathrm{e}$, d'altra parte, non pare che questa abilità sia data per scontata (è desumibile da alcune precisazioni linguistiche di cui si è detto). L'obiettivo del bordolese sembra invece un altro: acuire la sensibilità dei suoi allievi nel confronto linguistico e, dopo aver verificato la sostanziale parità delle due lingue e del contenuto dei rispettivi enunciati, confermare la validità del messaggio morale trasmesso dalle massime.

È importante precisare che il "code-switching", pur essendo qui connesso agli scopi della didattica, conserva anche la sua distintiva funzione sociale perché il Ludus, in un'ottica pedagogica, offre anche un modello di bilinguismo d'élite ${ }^{60}$ a cui i giovani dovevano abituarsi ${ }^{6}$. La traduzione latina, invece, ha una funzione insieme storica ed etica perché accosta al materiale di partenza greco citazioni dalla commedia e nozioni sulla storia della civiltà, inoltre la versione è la premessa per l'adattamento al costume e alla morale romana.

Evidentemente Ausonio si rendeva conto delle difficoltà di trasposizione da una lingua all'altra e dell'impossibilità di realizzare una perfetta sovrapposizione tra gli enunciati greci e romani, per ragioni linguistiche e culturali; percepiva che la diversità di dettato in alcune versioni era riflesso della differente sensibilità e, per questo, dotò le traduzioni di commenti e di interpretazioni. La spiegazione, però, non solo appiattiva lo spessore culturale che separava mondo greco e romano, ma, annullando pure la dimensione diacronica, non distingueva tra il pensiero greco arcaico, la commedia latina e le riflessioni etiche maturate fino al IV secolo d.C. ${ }^{62}$ La traduzione, insomma, veniva ad essere al servizio dell'istanza morale, obiettivo principale del Ludus: l'ammaestramento linguistico nella versione dal greco va di pari passo con la persuasione alla virtù ${ }^{63}$; l'importanza dei valori etici è comprovata proprio dalla presenza delle $\gamma v \tilde{\omega} \mu \alpha \mathrm{l}$ anche nella loro veste originale greca (fatto inusuale rispetto al panorama delle altre composizioni sui sette sapienti), nonché dal suo corrispettivo latino, a indicare la sostanziale unità della riflessione classica e delle sue categorie di giudizio. 


\section{BIBLIOGRAFIA}

ADAMS James Noel, Bilingualism and the Latin Language, Cambridge, Cambridge University Press, 2003.

ADAMS James Noel, «Romanitas and the Latin Language», Classical Quarterly, 53, 2003bis, p. 184-205.

ALTHOFF Jochen - ZELLER Dieter, Die Worte der Sieben Weisen, griechisch und deutsch. hrsg., übers. und komm. von A.J. und Z.D. Mit Beiträgen von ASPER Markus, Z.D. und SPAHLINGER Lothar, Darmstadt, WGB, 2006, «Texte zur Forschung» vol. 89.

AUWERS Jean-Marie - SOMERS Véronique, «Pèlerinage au cœur de soi-même ou variations patristiques sur "connais-toi toi-même“", in CASEAU Beatrice, CHEYNET Jean-Claude, DÉROCHE Vincent (éds.), Pèlerinages et lieux saints dans l'Antiquité et le Moyen Âge. Mélanges offerts à Pierre Maraval, Paris, Association des amis du Centre d'histoire et de civilisation de Byzance, 2006, «Travaux et mémoires du Centre de recherche d'histoire et civilisation de Byzance.

Monographies» vol. 23, pp. 1-12.

BELLINCIONI Maria, Educazione alla sapientia in Seneca, Brescia, Paideia, 1978, «Antichità Classica e Cristiana» vol. 17.

BELLINCIONI Maria, Lucio Anneo Seneca. Lettere a Lucilio. Libro XV: le lettere 94 e 95, Brescia, Paideia, 1979, «Testi classici» vol. 5.

BENEDETTI Fabrizio, La tecnica del "vertere" negli epigrammi di Ausonio, Firenze, L.S. Olschki, 1980, «Studi» vol. 56.

BENVENISTE Émile, «Latin tempus», in Mélanges de philologie, de littérature et d'histoire anciennes offerts à Alfred Ernout, Paris, C. Klincksieck, 1940, p. 11-16.

воотн Alan D., «The Academic Career of Ausonius», Phoenix, 36, 1982, p. 329-343.

BREITMEYER Jules, Le suffixe latin -ivus, Genève, Fax, 1933.

BRUNCO Wilhelm, «De dictis VII sapientum a Demetrio Phalereo collectis», Acta Seminarii

Erlangensis III, 1884, p. 299-398.

BURCK Erich, «Die Grundwerte der römischen Lebensordnung. Labor, moderatio, pietas», Gymnasium , 58, 1951, p. 161-183.

CARCATERRA Antonio, «L'analisi del ius e della lex in elementi primi: Celso, Ulpiano, Modestino», Studia et Documenta Historiae et Iuris, 46, 1980, p. 248-281.

CHAHOUD Anna, «Alterità linguistica, latinitas e ideologia tra Lucilio e Cicerone», in oNIGA Renato e VATTERONI Sergio, Plurilinguismo letterario, Soveria Mannelli (Catanzaro), Rubbettino, 2007, p. 41-58.

CoşKun Altay, Die gens Ausoniana an der Macht. Untersuchungen zu Decimus Magnus Ausonius und seiner Familie, Oxford, Unit for Prosopographical research (Linacre college), 2002, «P. \& G.» vol. 8. COURCElle Pierre, Connais-toi toi-même. De Socrate à Saint Bernard, Paris, Etudes augustiniennes, 1974, «Collection des etudes augustiniennes. Antiquité» vol. 58-59-60 (ed. italiana: Conosci te stesso. Da Socrate a san Bernardo, presentazione di REALE Giovanni, trad. it. di FILIPPI Francesca, 
Milano, Vita e Pensiero, 2001, «Pubblicazioni del Centro di ricerche di metafisica. Temi metafisici e problemi del pensiero antico» vol. 85).

DAUGE Yves Albert, Le barbare. Recherches sur la conception romaine de la barbarie et de la civilisation, Bruxelles, Latomus, 1981.

DIONISOTTI Anna Carlotta, «From Ausonius' Schooldays? A Schoolbook and Its Relatives», Journal of Roman Studies, 72, 1982, p. 83-125.

DUBUISSON Michel, «Barbares et barbarie dans le monde gréco-romain: du concept au slogan», L'Antiquité Classique, 70, 2001, p. 1-16.

ETIENNE Robert, Bordeaux antique, Bordeaux, [s.n.], 1962, «Histoire de Bordeaux» vol. 1.

FOCARDI Gabriella, «Linguaggio forense nei prologhi terenziani», Studi Italiani di Filologia Classica, 44,1972 , p. 55-88.

FOCARDI Gabriella, «Ambientazione forense e parodia nel Phormio di Terenzio», Sileno 16, 1990, p. 107-155.

FOLENA Gianfranco, Volgarizzare e tradurre, Torino, Giulio Einaudi Editore, 1991 (già apparso negli atti del convegno La traduzione, saggi e studi, Trieste 1973, 57-120).

FREYBURGER Gérard, «Le mot "barbarus" dans l'oeuvre de Ciceron», in Mélanges offerts a Léopold Sèdar Senghor. Langues - Littérature - Historie anciennes, Dakar, Les Nouvelles éditions Africaines, 1977, p. 141-152.

GoLDLUST Benjamin, «Le statut de la culture grecque dans la poétique d'Ausone», Latomus, 69, 2010, p. 129-149.

GREEN Roger P.H., «Greek in Late Roman Gaul. The Evidence of Ausonius», in CRAIK Elizabeth M., Owls to Athens. Essays on Classical Subjects presented to Sir K. J. Dover, Oxford, Clarendon Press, 1990, p. 311-319.

GREEN Roger P.H., The Works of Ausonius, Edited with Introduction and Commentary by. G.R.P.H., Oxford, Clarendon Press, 1991.

GREEN Roger P.H., Decimi Magni Ausoni Opera, recognovit brevique adnotatione critica instruxit G.R.P.H., Oxford, Clarendon Press, 1999, «Scriptorum classicorum bibliotheca Oxoniensis».

HANSLIK Rudolf, «Pacatus 2», RE XVII (2) 1942, 2058-2060.

HERZOG Reinhart - SCHMIDT Peter Lebrecht, Nouvelle histoire de la littérature latine, vol. IV: L'âge de transition. De la littérature romaine à la littérature chrétienne de 117 à 284 après J.-C., editée par SALLMANN Klaus, version française sous la direction de HEIM François, Turnhout, Brepols, 2000. HOPKINS Morris Keith, «Social Mobility in the Later Roman Empire: the Evidence of Ausonius», Classical Quarterly, 11, 1961, p. 239-249.

JONES Arnold Hugh Martin - MARTINDALE John Robert - MORRIS John, The Prosopography of the Later Roman Empire, Cambridge, Cambridge University Press, 1971.

KAIMIO Jorma, The Romans and the Greek Language, Ekenäs, Ekenäs Tryckeri Aktiebolag, 1979, «Commentationes Humanarum Litterarum» vol. 64.

KASTER Robert A., Guardians of Language: the Grammarian and Society in Late Antiquity, Berkeley, University of California Press, 1988, «The transformation of classical heritage» vol. 11. KOSTER Severin, «Vir bonus et sapiens. Ausonius 363 p. 90 P.», Hermes 102, 1974, p. 590-619. 
KRAMER Johannes, Glossaria bilinguia altera (C. Gloss. Biling. II), München und Leipzig, Saur, 2001, «Archiv für Papyrusforschung und verwandte Gebiete. Beiheft» vol. 8.

LEUMANN Manu, Lateinische Laut- und Formenlehre, München, C.H.Beck, 1977.

LOSSAU Manfred Joachim, «Ausonius und litterae Graecae», Maia, 41, 1989, p. 125-142.

LOYEN André, Sidoine Apollinaire. Tome III: Lettres (livres VI-IX), texte établi et traduit par A.L., Paris, Les Belles Lettres, 1970.

MARRAMAo Giacomo, Kairós. Apologia del tempo debito, Bari, Laterza, 1992, «Sagittari Laterza» vol. 60.

MASSIONI Marina, «L'evocazione forense nel Phormio di Terenzio», Sileno, 19, 1993, p. 159-177.

MONDIN Luca, Decimo Magno Ausonio. Epistole. Introduzione, testo critico e commento, Venezia, Il Cardo, 1995.

MUNARI Franco, «Ausonio e gli epigrammi greci», Studi Italiani di Filologia Classica, 28, 1956, p. 308-314.

NEWMAN Robert J., "Cotidie meditare. Theory and Practice of the meditatio in Imperial Stocism», ANRW 36.3, 1989, p. 1473-1517.

ONIANS Richard Broxton, The Origins of European Thought about the Body, the Mind, the Soul, the World, Time and Fate. New Interpretations of Greek, Roman and kindred evidence also of some basic Jewish and Christian Beliefs, Cambridge, Cambridge University Press, 1989 (1951 ${ }^{1}$, trad. it. Le origini del pensiero europeo intorno al corpo, la mente, l'anima, il mondo, il tempo e il destino. Nuove interpretazioni di materiali greci e romani, di altre testimonianze e di alcune fondamentali concezioni ebraiche e cristiane, a c. di PERILli Lorenzo, trad. di ZANINONI Paolo, Milano, Adelphi, 1998, «Il ramo d'oro» vol. 31). отто August, Die Sprichwörter und sprichwörtlichen Redensarten der Römer, Leipzig, 1890.

PARATORE Ettore, «Letteratura pagana nella Gallia romana», in Atti del colloquio sul tema: la Gallia romana. Roma 10-11 maggio 1971, Roma, Accademia Nazionale dei Lincei, 1973, «Problemi attuali di scienza e di cultura» vol. 158, p. 53-86.

PEIPER Rudolph, Decimi Magni Ausonii Burdigalensis Opuscula, Lipsia, Teubner, 1886, «Bibliotheca scriptorum Graecorum et Romanorum Teubneriana».

PICHON René, Les derniers écrivains profanes: les panégyristes, Ausone, le "Querolus", Rutilius Namatianus , Paris, Leroux, 1906, «Etudes sur l'histoire de la littérature latin dans les Gaules».

RAVEnNa Giovanni, Le nozze di Polemio e Araneola (Sidonio Apollinare, Carmina XIV-XV), Bologna, Patron, 1990, «Testi e manuali per l'insegnamento universitario del latino» vol. 33.

ROCHETTE Bruno, «Du grec au latin et du latin au grec. Les problèmes de la traduction dans l'antiquité gréco-latine», Latomus, 54, 1995, p. 245-261.

ROCHETTE Bruno, «Code-Switching chez Ausone», in BEDON Robert \& POLFER Michel (éds.), Être Romain. Hommages in memoriam Charles Marie Ternes, Remshalden, Greiner, 2007, p. 175-195. ROCHETTE Bruno, «Grecs, Romains et Barbares. À la recherche de l'identité ethnique et linguistique des Grecs et des Romains», Revue Belge de Philologie et d'Histoire, 75, 1997, p. 37-57. ROGER Maurice, L'enseignement des lettres classiques d'Ausone à Alcuin: Introduction à l'histoire des écoles carolingiennes, Paris, A. Picard et fils, 1905. 
SCHEIDLE Kurt, Modus optumum. Die Bedeutung des »rechten Maßes« in der römischen Literatur (Republik - frühe Kaiserzeit), untersucht an den Begriffen »Modus - Modestia - Moderatio - Temperantia», Frankfurt am Main, P. Lang, 1993, «Studien zur klassischen Philologie» vol. 73.

schenkl Carl, D. Magni Ausonii Opuscula, Berlino, Weidmann, 1883 «MGH.AA.» 5.2.

SCHWAB Andreas, Thales von Milet: Darstellungen seiner Figur und seiner Ideen in den griechischen und lateinischen Textzeugnissen christlicher Autoren des 2.-6. Jh. n. Chr., Berlino, De Gruyter, 2011, «Studia Praesocratica» vol. 3.

SIVAN Hagith, Ausonius of Bordeaux. Genesis of a Gallic Aristocracy, London, Routledge, 1993.

SPITZER Leo, L'armonia del mondo. Storia semantica di un'idea, Bologna, Il Mulino, 2006 ( $1^{\text {a }}$ ed. it. Bologna 1967; ed. orig. Classical and Christian Ideas of World Harmony, Baltimore, John Hopkins Press, 1963).

STAHL Ferdinand, De Ausonianiis studiis poetarum Graecorum, Kiliae, ex officina C.F. Mohr, 1886. TERNES Charles-Marie, «La sagesse grecque dans l'œuvre d'Ausone», Comptes-rendus de l'Académie des inscriptions et belles-lettres, 130, 1986, p. 147-161.

TOSI Renzo, Dizionario delle sentenze latine e greche, Milano, Rizzoli, 1991 (traduzione francese: Dictionnaire des sentences latines et grecques, Grenoble, Jérôme Million, 2010).

TRAINA Alfonso, «Su Ausonio “traduttore”», Rivista di Filologia e di Istruzione Classica, 110, 1982, p. 111-115.

TRAINA Alfonso, «Semantica del carpe diem», in Poeti latini (e neolatini). Note e saggi filologici, Bologna, Pàtron, $1986^{2}$, p. 227-251 (= Rivista di Filologia e Istruzione Classica, 101, 1973, p. 5-21). TRAINA Alfonso, «Le traduzioni», in CAVALlo Guglielmo, FEDELI Paolo, GIARDINA Andrea, Lo spazio letterario di Roma antica, vol. II: La circolazione del testo, Roma, Salerno Editrice, 1989, 93-123. TURCAN - VERKERK Anne-Marie, Un poète latin chrétien redecouvert: Latinius Pacatus Drepanius, panégyriste de Théodose, Bruxelles, Éditions Latomus, 2003, «Collection Latomus» 276.

TZIATZI PAPAGIANNI Maria, Die Sprüche der sieben Weisen: zwei byzantinische Sammlungen. Einleitung, Text, und Kommentar, Stuttgart, Teubner, 1994, «Beiträge zur Altertumskunde» vol. 51.

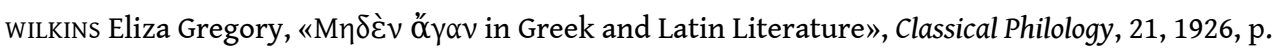
132-148.

\section{NOTE}

1. Una prima versione di questo lavoro è stata presentata oralmente nel 2011 nel corso della $X$ edizione della European Summer School of Classics di Trieste (con il titolo Gnothi seauton, quod Latinum est 'nosce te'. Massime sapienziali greche e valori etici romani nel Ludus septem sapientum di Ausonio). L'articolo che Rursus qui accoglie è stato sottoposto alla redazione nel 2012; i materiali in esso contenuti sono confluiti in veste definitiva nell'edizione Decimi Magni Ausonii Ludus septem sapientum. Introduzione, testo, traduzione e commento a cura di Elena Cazzuffi. Hildesheim et al.: Olms 2014 (Spudasmata 160). Ringrazio i professori Cristante e Fernandelli per avermi invitato a partecipare ai lavori della ESSC, il prof. Marangoni per i consigli e ricordo con gratitudine il prof. Schievenin che con lui ha sempre seguito il mio lavoro.

2. prof. 8,13ss. obstitit nostrae quia credo, mentis / tardior sensus neque disciplinis / appulit Gracis puerilis aevi / noxius error (cf. Aug. conf. 1,14,23). 
3. Ausonio raccomanda a suo nipote la lettura di Omero e di Menandro (protrept. 45ss.) e, in epist. 8,25-34, vanta una biblioteca di opere greche di tutto rispetto; sulla cultura greca del bordolese si vedano STAHL 1886, TERNES 1986, GREEN 1990 e GOLDLUST 2010.

4. Sono stati particolarmente studiati in questo senso gli epigrammi: vd. MUNARI 1956, BENEDETTI 1980, TRAINA 1982, LOSSAU 1989.

5. Cf. epist. 12 e vd. MONDIN 1995: 168s.

6. Cf. epiced. 9s. e Par. 3,15s.

7. La carriera accademica di Ausonio è percorsa da HOPKINS 1961: 242ss., воOTH 1982: 333ss., KASTER 1988: 247, SIVAN 1993: 59, 101 e COşKUN 2002: 34ss.

8. Il dato è ricavabile da un glossario bilingue greco-latino edito da Conrad Celtis nel $1495 \mathrm{e}$ redatto in Gallia in un periodo incerto (DIONISOTTI 1982: 123 propone la fine del III e l'inizio del IV secolo d.c.); tra gli autori studiati si citano Omero, Teocrito, Tucidide, Demostene, Ippocrate, Senofonte, dei non meglio precisati cinici e tres comoedias (su questo manuale nel panorama degli hermeneumata, vd. HERZOG-SCHMIDT 2000: 273 § 439.4 e KRAMER 2001: 15s.).

9. Cf. prof. 8,5s. sedulum cunctis studium docendi, / fructus exilis tenuisque sermo.

10. Il giudizio di Paratore è drastico: il Ludus non sarebbe altro che l'«ennesima variazione dei componimenti riassuntivi di tipo enciclopedico», un «lusus banale e bambinescamente esteriore» (PARATORE 1973: 75).

11. Personaggio emergente nella compagine politica della Gallia, Latino (o Latinio) Pacato Drepanio fu ministro delle province galliche, panegirista imperiale, proconsole d'Africa e comes rerum privatarum (vd. HANSLIK 1942: 2058-2060, JONES et alii 1971: 272, cOşKUN 2002: 96s. e 208 e TURCAN-VERKERK 2003).

12. Il Ludus compone varie informazioni utili per una lezione: dati sul mondo greco (vv. 24s. e 29s.), sul teatro romano (vv. 26-41), citazioni dai comici latini (e.g. vv. 131s., 155, 206) e, ovviamente, ragguagli sui sette sapienti e sulle $\gamma v \tilde{\omega} \mu \alpha 1$.

13. Di tale avviso ROGER 1905: 17, PICHON 1906: 156s. e ETIENNE 1962: 259.

14. Vd. TERNES 1986: 148ss. e cf. KOSTER 1974.

15. Non a caso le biografie e i detti dei sette preludono alla trattazione di storia della filosofia sia nell'opera di Diogene Laerzio (1,30ss.) sia in quella di Clemente Alessandrino (Strom. 1,14,59ss.), secondo una sistemazione del sapere di ascendenza aristotelica: in un frammento del Пعрі̀ $\varphi \imath \lambda о \sigma o \varphi i ́ \alpha \varsigma$ la saggezza politica dei sapienti rappresenta la fase immediatamente precedente

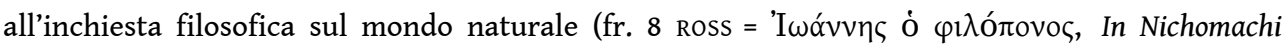
arithm. eisagogen 1,8,-2,42 HOCHE, VI sec. d.C.).

16. La circolazione di aneddoti e liste di nomi e di $\gamma v \tilde{\omega} \mu \alpha$ l dei sette sapienti è documentata incessantemente dall'antichità al Medioevo e oltre, sia in area orientale sia in area occidentale; il tema fu molto produttivo in letteratura, nelle arti visive e nell'epigrafia giacché si prestava a facili declinazioni in virtù della sua peculiare natura catalogica.

17. BRUNCO 1884 ne riproduce il testo e ne illustra il percorso nella tradizione orientale e occidentale; per una sintesi vd. il più recente ALTHOFF-ZELLER 2006: 10-12.

18. Vd. TZIATZI PAPAGIANNI 1994: 446.

19. TZIATZI PAPAGIANNI 1994: 435-438 indica tutti i mss. in cui l'epigramma compare; da esso dipende la traduzione latina De septem sapientibus ex Graeco, apparsa per la prima volta nell'edizione degli Opuscula ausoniani curata dall'Ugoleto che, verosimilmente, ne fu l'autore (cf. SCHENKL 1883: XXX, PEIPER 1886: 409 e GREEN 1991: 674).

20. Per la catena di versi, parte dell'epitalamio, si veda RAVENNA 1990: 66s.

21. Le tre sequenze sono state studiate, ponendo particolare attenzione alla figura di Talete, da SCHWAB 2011: 312-323. 
22. Ad esempio il Ludius enuncia in greco il detto di Solone e lo traduce (56s.); a sua volta Solone, nella sezione a lui dedicata, ripete la sentenza in greco (85) e ne dà due traduzioni latine (87 e 103).

23. I due comici con le loro opere sono ricordati a più riprese: vd. e.g. lud. 131s. (citazione combinata da Plaut. Men. 882s. e Ter. Phorm. 462), lud. 155 (Afer poeta vester 'ut ne quid nimis'), lud. 191 (citazione da Ter. Andr. 68), lud. 207 (vester quoque ille comicus Terentius), lud. 219 (citazione da Ter. Phorm. 214ss.), lud. 220 (meditanda cunctis comicus Terentius).

24. Vi sono alcune divergenze rispetto all'edizione di GREEN 1999: al v. 56 le cruces apposte a introfertur paiono allarmistiche a fronte dell'univoca paradosi e della lingua esibita nel Ludus, modellata sulla commedia arcaica dove le formazioni con intro sono ricorrenti; al 57 accogliamo la lezione qui di V a discapito del quo di PH (Green) che sembra una normalizzazione della forma arcaica; ai vv. 65 e 66 seguiamo il testo tradito Periandri est e meditationem esse contro l'edizione oxoniense est Periandri e esse meditationem, che con l'inversione dell'ordine cerca di risolvere lo iato in cesura, fenomeno che, tuttavia, rientra tra le scelte metriche di Ausonio; dicit al v. 67 è ancora lezione manoscritta rispetto al dixit proposto da Vinet e accolto da Green.

25. Accogliamo la paradosi, mantenuta da SCHENKL 1883 e da PEIPER 1886, rispetto alla variazione di GREEN 1999: quod est Latinum.

26. Interpretor è l'unica espressione che rientra propriamente nella terminologia della traduzione, di cui il latino è particolarmente ricco (vd. TRAINA 1989: 96s., ROCHETTE 1995: 249 e FOLENA 1991: 6s. e 80s. con un percorso dall'antichità classica all'Umanesimo).

27. Il resoconto latino dell'incontro con Creso non è verosimilmente una traduzione dall'originale greco: oltre a differenze marginali (come la sostituzione di Aglao a Cleobi e Bitone), vi è una notevole riduzione o meglio una sutura tra due parti molto distanti del logos lido, vale a dire l'abboccamento tra Creso e Solone (Hdt. 1,30-33) e quello tra Creso e Ciro (Hdt. 1,86-90), in funzione della dimostrazione di veridicità dell'assunto iniziale, ovvero dell'apoftegma soloniano. È plausibile che il bordolese abbia attinto da una fonte intermedia, probabilmente da una compilazione scolastica, o che si sia avvalso della consulenza di Staphylius di Auch, esaltato come conoscitore di Erodoto in prof. 20,8 historiam callens Livii et Herodoti.

28. Graece coactum est codd. (SCHENKL 1883, PEIPER 1886), est om. Scal. (GREEN 1999).

29. La traduzione del Ludius era stata più puntuale: finem intueri longae vitae qui iubes (lud. 57).

30. Ancipiti statu è una variazione della più frequente iunctura tra anceps e eventum (cf. e.g. Liv. 4,27,6; Val. Max. 1,8,6; Amm. 31,7,9 - 31,9,1).

31. ThlL V.2 77,19ss. interpreta lud. 86 come disserere ..., explanare, interpretari; e, infatti, al v. 121 occorre con il significato di 'esporre' (seriem per omnem cuncta regi edisserit).

32. Edissertator è un'intuizione dell'Ugoleto sulla base delle lezioni et dissertator (V), edessertator (P) e edesserator $(\mathrm{H})$; nomen agentis deverbativo da edisserto, frequentativo di edissero, è hapax di conio ausoniano (cf. ThlL V.2 78,39-41 s.v. edissertator [Köster - Mann]) derivato dall'uso plautino del verbo edisserto (Amph. 600, Cas. 915, Stich. 302).

33. Sul "conosci te stesso" vd. courCelle 1974: vol. 1,117ss. (= COURCELLE 2001: 108ss.) e, con preciso riferimento alla produzione patristica, vd. AUWERS-SOMERS 2006: 1-12.

34. Così in hered. 17ss. verum ager iste meus quantus sit, nosce, etiam ut me / noveris et noris te quoque,

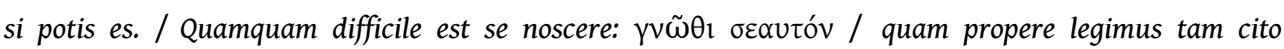
neglegimus e in ecl. 20,13s. non prius in dulcem declinans lumina somnum / omnia quam longi reputaverit acta diei (cf. KOSTER 1974: 590ss. e TERNES 1986: 150s.).

35. La precisazione è assente nelle altre composizioni catalogiche sui detti dei sette sapienti: Hyg. fab. 221,2 ex Ephyre Periandre doces, cuncta emeditanda, Sidon. carm. 2,159 ex Efyra totum meditaris quod Periander e 15,46 tu meditans totum decoras, Periander, Corinthon, Lux. anth. 351,12 $\mathrm{R}^{2}$ omnia constituit tecum ut meditando revolvas. 
36. Segnaliamo altre difformità rispetto all'edizione oxoniense: al v. 215 seguiamo l'univoca lezione ms. contro l'inserzione di iam dopo dictum finalizzata all'eliminazione dello iato (add. PEIPER 1886, GREEN 1999); al v. 216 ci atteniamo ancora al testo tradito rispetto al meditationem esse omne quod recte geras di GREEN 1999; al v. 221 optiamo per sedes, lezione ms., a fronte dell'aedes scelto dall'editore per ragioni di significato, ma privo di motivazioni paleografiche e metriche; infine al v. 227 anticipiamo la pausa di senso con un segno di punteggiatura tra sit e dehinc, mentre GREEN 1999 lo colloca dopo dehinc.

37. Vi sono evidenti punti di contatto con gli ammonimenti a Lucilio dell'epistolario senecano dove la meditatio non deve avere effetto solo sul mondo interiore del soggetto "meditante", ma deve riflettersi anche sulle sue azioni perché lo scopo della filosofia è ispirare il sapiens ad agire rettamente in ogni circostanza (epist. 85,32 huic [scil. sapienti] enim propositum est in vita agenda non utique, quod temptat, efficere, sed omnia recte facere); ovvia premessa per il raggiungimento di un tale obiettivo è che la riflessione anticipi l'azione: in duas partes virtus dividitur, in contemplationem veri et actionem (Sen. epist. 94,45, su cui vd. NEWMAN 1989: 1483, BELLINCIONI 1978: 103ss. e EAD. 1979: 178).

38. Per le attestazioni si vedano отто 1890, s.v. modus e TOSI 1991: 785s. (tra le numerose, si ricorda Hor. sat. 1,1,106 est modus in rebus ...).

39. BURCK 1951: 167ss.

40. La documentazione, piuttosto ricca ed estesa cronologicamente, è stata raccolta da SCHEIDLE 1993: 35-48 e 54, ma vd. anche WILKINS 1926: 144ss.

41. Hyg. fab. 221,9 Milesiusque Thales sponsori damna minatur, Sidon. carm. 2,157 Mileto quod crete Thales vadimonia culpas e 15,44 Thales Mileto genius vadimonia damnat; Lux. anth. 351,7-8 $\mathrm{R}^{2}$ ascrive a Talete un'altra massima: Thales ingenio sapiens Milesius acri / errorem in terris firmat non caelitus esse. 42. Vd. FOCARDI 1990: 110ss. e MASSIONI 1993: 165s.

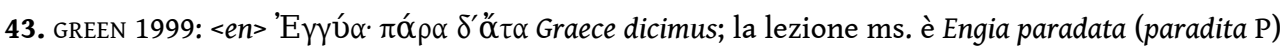
ecce dicimus. Il verso è stato rimaneggiato in due punti dagli editori: in sede incipitaria (nos Scaligero, en SCHENKL 1883, GREEN 1999) e in posizione intermedia con la sostituzione di ecce con Graece (PEIPER 1886). Accogliamo quest'ultima proposta perché sembra armonizzarsi con le altre marche traduttive presenti nel Ludus (cf. vv. 85,86,190).

44. Hyg. fab. 221,4 plures esse malos, Sidon. carm. 2,161 Prienaee Bia, quod plus tibi turba malorum est e Lux. anth. 351,10R2 plures esse malos, con l'unica variante del sidoniano carm. 15,48 Prienaee Bia, plures ais esse malignos che, rende kakó con malignus e ne accentua l'aspetto dell'inclinazione alla malvagità.

45. ROCHETTE 1997: 43ss. e soprattutto DUBUISSON 2001: 4.

46. Vd. e.g. Caes. Gall. 1,40,9 - 1,44,9 - 4,22,1 - 6,10,2 e Cic. orat. 1,118 - 3,223, per tutti si rinvia al commento di DAUGE 1981: 106 n. 117 e 124 n. 168.

47. L'analisi componenziale di NDIAYE 2005: 133 vi include i seguenti semi: +animato / +umano / +straniero / +senza statuto istituzionale / +che parla una lingua che non si comprende / +né romano né greco / +nemico / +caratterizzato dalla feritas / +caratterizzato da vanitas / +peggiorativo.

48. Cf. Cels. dig. 1,1,1 ius est ars boni et aequi; questa definizione scompone in elementi primi il concetto di ius, cioè la norma intesa estensivamente anche nella sua applicazione, che deve ottemperare alle due finalità di "idoneità" (il bonum, la capacità di raggiungere gli scopi prefissati) e di "ponderazione delle situazioni e di opposti interessi" (l'aequum, sul quale vd. CARCATERRA 1980: 258).

49. Anche DAUGE 1980: 570 è convinto che Ausonio ironizzi.

50. GREEN 1999: Mitylena <ego> ortus Pittacus sum Lesbius; ego è inserzione del Mertens per evitare lo iato con il successivo ortus. 
51. Per una svista la frase citata è riferita alla virgo Antifila, mentre in realtà nella commedia eam intendeva la meretrix Bacchide; la memoria di Ausonio fallisce ancora perché nell' Heautontimoroumenos è il servo Siro a pronunciarla e non Dromone.

52. BENVENISTE 1940: 13, ONIANS 1989: 344, MARRAMAO 1992: 98s., SPITZER 2006: 89s.

53. In origine termine tecnico dell'agricoltura (LEUMANN 1977: 304 § 281c) e della religione pagana, tempestivus divenne d'uso assai frequente grazie al considerevole ruolo rivestito nella vita romana dalla consultazione degli oracoli e dall'interpretazione dei segni; di là, per estensione di senso, sarà stato impiegato tutte le volte che si trattava di esprimere il concetto id "opportuno", di "favorevole", senza che il sostantivo a cui veniva applicato si riferisse necessariamente al tempo o rappresentasse una nozione legata alla vita dei campi o all'allevamento di animali (BREITMEYER 1933: 259).

54. La figura, in linea con l'etimologia antica (Varro ling. 6,3 e cf. GLOSS. IV Plac. T 8), serve a ottenere una più precisa traduzione di kaıpós.

55. Vd. BENVENISTE 1940: 14s.

56. Questa "etichetta" sociolinguistica, che definisce l'alternanza di codice entro uno stesso enunciato, è stata applicata alla produzione ausoniana da ROCHETTE 2007: 175ss. (per il Ludus, trattato cursoriamente, vd. p. 191s.).

57. In questi casi si può parlare di "mixed-language text", perché greco e latino non sono giustapposti, ma si fondono a formare un discorso unitario; se le due lingue invece fossero contrapposte e fisicamente separate e il loro contenuto fosse almeno in parte sovrapponibile, si dovrebbe parlare di testi bilingui (ADAMS 2003: 30 e 67).

58. I tre tipi di code-swiching, tag-switching, inter-sentential switch, intra-sentential switch, sono descritti con particolare riferimento al latino da ADAMS 2003: 21-24.

59. La traduzione era un esercizio di stile diffuso nelle scuole romane (tra gli altri vd. CLARKE 1957: 170s., KAIMIO 1979: 201, ROCHETTE 1995: 257).

60. Sul bilinguismo d'élite in ambito romano vd. ADAMS 2003: 9.

61. Nella tarda antichità il greco era divenuto un distintivo socio-culturale tanto più potente quanto più, su ampia scala, se ne era persa la padronanza.

62. Si tratta di una dinamica costitutiva la tradizione linguistico-culturale romana, dove «la nozione del tradurre assume un'importanza fondamentale ... nel collegamento diacronico e nell'attualizzazione linguistica di valori del passato in una simbiosi (e tensione) fra moderno e antico che anche nella traduzione si ripresenterà con l'umanesimo greco-latino (valore diacronico-attualizzante della traduzione)» (FOLENA 1991, 7s.).

63. Fa parte della tradizione latina, da Lucilio a Cicerone, l'idea che le scelte linguistiche riflettano precise inclinazioni morali e che l'identità di un vero civis romanus dipenda anche dalla sua competenza linguistica (ADAMS 2003bis: 185-188, CHAHOUD 2007: 41-45 e 51-55).

\section{RIASSUNTI}

Il Ludus septem sapientum, operetta in senari giambici sulle $\gamma v \tilde{\omega} \mu \alpha \iota$ dei sette sapienti, rientra fra gli opuscula ausoniani di ispirazione scolastico-sapienziale; tuttavia alla tradizionale interpretazione dell'opera come composizione pedagogica va applicato il seguente correttivo: l'apprendimento del greco non è il primo e l'unico obiettivo didattico che il Ludus si prefigge, 
perché la traduzione, spesso porta in modo problematico, serve a sviluppare una riflessione sulle differenze linguistiche, culturali ed etiche tra mondo greco e romano.

Ausonius' Ludus septem sapientum is a sort of drama about the seven wise men's $\gamma v \tilde{\omega} \mu \alpha \mathrm{l}$; it belongs to a group of opuscula dealing with school and wisdom. The work has often been interpreted as a didactic composition and some scholars thought that the teaching of the Greek language was its main aim. On the contrary we believe the Greek learning is not the poet's chief object; in fact the opusculum does not offer any element of Greek grammar, indeed it compares the $\gamma v \tilde{\omega} \mu \alpha \mathrm{l}$ and their Latin translations in order to develop a reflection upon differences in Hellenic and Roman languages, cultures, and ethics.

INDICE

Keywords : Ausonius, Ludus, Sententia, Translation, Seven Wise Men

\section{AUTORE}

\section{ELENA CAZZUFFI}

ecazzuffi@gmail.com

Assegnista di ricerca presso il Dipartimento di Scienze Storiche, Geografiche e dell'Antichità dell'Università degli Studi di Padova; nel 2010 ha conseguito il diploma di dottore di ricerca in Filologia Classica discutendo una tesi sul Ludus septem sapientum ausoniano. I suoi studi sono rivolti principalmente al Tardoantico, ad Ausonio e a Claudiano. 